\title{
Journal of Membrane Biology: Biophysics
}

\author{
Sandro Keller ${ }^{1}$ Ana-Nicoleta Bondar ${ }^{2} \cdot$ Jana Broecker $^{3}$ • \\ Alexey S. Ladokhin ${ }^{4} \cdot$ Eva-Kathrin Sinner ${ }^{5}$
}

Published online: 20 April 2016

(c) Springer Science+Business Media New York 2016

\section{Section: Biophysics}

Great news for all membrane biophysicists: the Journal of Membrane Biology (JMBi) now features a new section devoted to Biophysics! Biological membranes display a bewildering degree of complexity, diversity, and heterogeneity at all levels, thus providing an exceedingly fascinating and challenging research subject for different, overlapping scientific disciplines. Singling out a particular membrane component or property suspected to be involved in a biological process is often difficult or even impossible. Simplifying approaches and assumptions thus become unavoidable if one is to understand the structures, dynamics, and functions of biological membranes in greater detail and in a quantitative manner. Such simplifications come in different flavors, ranging from model membrane systems of reduced complexity to concepts that help disentangle interrelated phenomena into manageable pieces. Both of these examples fall into the realm of biophysics, and numerous other concepts originating from seemingly remote branches of physics have proven their great value in the study of biological membranes. Prominent examples include phenomenological treatments of membranes as capacitors, semipermeable barriers, or bilayer couples and, at the other extreme, atomistic simulations relying on molecular dynamics and quantum mechanics.

It is in this spirit that we encourage prospective authors to contribute papers that address pressing questions in the biomembrane field with the aid of concepts rooted in physics and physical chemistry. Hence, the scope of the Biophysics section shall go beyond the mere application of so-called biophysical techniques to membranes, although such submissions are certainly welcome if they provide novel, quantitative insights into the physical principles underlying the properties of and the processes taking place at and within membrane systems. This is meant to include not only genuine biological membranes but also the various artificial membrane mimetics that are often used in the laboratory or individual membrane constituents such as lipids and proteins.

We are excited to hear about your opinion on the reorganized structure of $J M B i$ and look forward to receiving your high-quality contributions to the new Biophysics section.
Sandro Keller

mail@sandrokeller.com

1 University of Kaiserslautern, Kaiserslautern, Germany

2 Freie Universität Berlin, Berlin, Germany

3 University of Toronto, Toronto, Canada

4 University of Kansas Medical Center, Kansas City, KS, USA

5 University of Natural Resources and Life Sciences, Vienna, Austria 